\title{
Species separation in polystyrene shock release evidenced by molecular-dynamics simulations and laser-drive experiments
}

\author{
Shuai Zhang $\odot,{ }^{1, *}$ D. E. Fratanduono, ${ }^{2, \dagger}$ M. C. Marshall $\odot,{ }^{1}$ J. R. Rygg,${ }^{1,3,4, \$}$ Amy E. Lazicki, ${ }^{2}$ A. Shvydky, ${ }^{1}$ \\ D. Haberberger, ${ }^{1}$ V. N. Goncharov, ${ }^{1}$ T. R. Boehly, ${ }^{1}$ G. W. Collins,,${ }^{1,3,4}$ and S. X. Hu $\oplus^{1,3,8}$ \\ ${ }^{1}$ Laboratory for Laser Energetics, University of Rochester, Rochester, New York 14623, USA \\ ${ }^{2}$ Lawrence Livermore National Laboratory, Livermore, California 94550, USA \\ ${ }^{3}$ Department of Mechanical Engineering, University of Rochester, Rochester, New York 14623, USA \\ ${ }^{4}$ Department of Physics and Astronomy, University of Rochester, Rochester, New York 14623, USA
}

(Received 20 August 2021; accepted 12 January 2022; published 17 February 2022)

\begin{abstract}
Material shock release generally happens in the targets of high-energy-density (HED) and inertial confinement fusion (ICF) experiments but has been challenging to study experimentally, theoretically, or computationally. Here, we report extensive studies of polystyrene $(\mathrm{CH})$ shock release by employing large-scale nonequilibrium molecular dynamics and laser-drive experiments at various shock strengths. Our experimental design prevents radiation preheating of the sample and employs a witness foil to investigate the release of shocked $\mathrm{CH}$ across a vacuum gap. We observe earlier acceleration of the foil by the release of $\mathrm{CH}$ under stronger shocks as well as reflectivity changes in the interferometry data before the foil moves, which is strong evidence of hydrogen streaming ahead of carbon at the release front, consistent with findings from our simulations. Furthermore, our calculations show that lighter species or hydrogen isotopes can carry more mass by one to two orders of magnitude to farther distances during the release and that only less than 0.1 times thermal expansion as predicted by hydrodynamics is needed to explain the high velocities and large scale lengths of low-density plasmas observed in radiation-preheated $\mathrm{CH}$ release experiments. These results highlight the significant role of species separation in the shock release of compounds. This process shall be considered, and its potential effects shall be clarified, in the design, interpretation, and analysis of future HED and ICF experiments.
\end{abstract}

DOI: 10.1103/PhysRevResearch.4.013126

\section{INTRODUCTION}

The shock release of matter into vacuum or low-density gases is of great interest to those researching inertial confinement fusion (ICF), high-energy-density (HED) sciences [1-4], planetary sciences [5], and astrophysics [6] but is challenging to study both experimentally and theoretically. Experimental investigations often rely on single-component hydrodynamic simulations as a regular practice, which use the Navier-Stokes equations that are based on a continuum fluid picture of flux [7]. The exclusion of atom-specific properties and chemical changes causes problems (such as kinetic effects [8-17]) for target design, performance, and even interpretation of experiments. This calls for a better understanding of shock release from a variety of matter in broad ranges of pressures, temperatures, and space and time scales.

As a common ablator material, polystyrene $(\mathrm{CH})$ is heavily used in and of critical importance to ICF and HED

\footnotetext{
*szha@lle.rochester.edu

†fratanduono1@1lnl.gov

†ryg@1le.rochester.edu

§shu@1le.rochester.edu
}

Published by the American Physical Society under the terms of the Creative Commons Attribution 4.0 International license. Further distribution of this work must maintain attribution to the author(s) and the published article's title, journal citation, and DOI. experiments $[18,19]$. Its equation of state (EOS) has been extensively studied and well understood with an experimentally measured Hugoniot to gigabar $\left(1 \mathrm{Gbar}=10^{5} \mathrm{GPa}\right)$ pressures [20-34], but the shock-release properties and their roles in affecting target performance are still poorly understood as shown by recent experiments [35,36]. Ross et al. observed separation of hydrogen from carbon on the laser-ablated side of $\mathrm{CH}_{2}$ using Thomson scattering techniques [35]. Haberberger et al. used optical interferometry to measure the temporal evolution of shock-released plasmas from the rear side of $\mathrm{CH}$, and the results show a large discrepancy from predictions by hydrodynamic simulations that ignore radiation effects [36]. Interestingly, the simulations and experiments agree when considering radiation transport, which renders preexpansion at the rear surface of $\mathrm{CH}$ before shock arrival [36-38]. This has motivated us to explore approaches that are beyond hydrodynamics to better simulate the shock release of $\mathrm{CH}$.

Recently, by successfully pushing large-scale nonequilibrium classical molecular-dynamics (CMD) simulations to over $2000 \mathrm{GPa}$, Zhang and Hu showed the occurrence of species separation upon breakout of a strong shock from $\mathrm{CH}$ [39]. The simulations showed that carbon lagged behind hydrogen, which forms low-density plasmas whose velocities and scale lengths both match experiments [36,39]. In another work, Stanton et al. applied CMD simulations to study mixing at a heated polystyrene-deuterium-tritium (CH-DT) interface [40]. Earlier theoretical work and particle-in-cell 
simulations by Amendt et al. also suggested charge separation in multispecies plasmas driven by barodiffusion (or pressuregradient-driven diffusion) and electric fields, phenomena that are not modeled in mainline single-fluid simulations [41-44]. These studies have demonstrated novel microscopic physics yet to be discovered from compounds and layered systems and for applications in ICF and HED experiments. Such microscopic physics can be understood by using CMD simulations that fully account for motion at the atomic level that are not considered in continuum models including hydrodynamics.

Aside from the widespread interest in hot plasma and strong shock research, the microscopic physics in materials with multiple species and the differences from hydrodynamic descriptions can also be pivotal to HED and ICF research at megabar $\left(1 \mathrm{Mbar}=10^{2} \mathrm{GPa}\right)$ or lower pressures. Materials shock compressed to such conditions can have chemical bonds that dissociate with increasing temperature $[31,45]$, while molecules and atomic clusters can persist in the subsequent expanding release. A self-consistent description of the shock release requires large simulation cells that are beyond the typical application regimes of first-principles methods but more suitable for CMD, although challenges remain in defining appropriate interactions among partially ionized species. Carefully designed computational and experimental research on $\mathrm{CH}$ at such pressures not only will be useful for its application in ICF and HED experiments, but also can motivate future studies on other materials or more complicated systems.

In this paper, we extend the CMD simulation efforts to study the release of shocked $\mathrm{CH}$ by considering a broad range of shock strengths, multiple types of hydrogen isotopes, and different degrees of thermal expansion of the $\mathrm{CH}$ sample caused by radiation preheat, which are relevant to various ICF and HED campaigns [37,38,46-48]. Our calculated postshock properties of $\mathrm{CH}$ are consistent with experimental and first-principles Hugoniots in a broad range of pressures $(\sim 50$ $13500 \mathrm{GPa}$ ), and our computational predictions of the $\mathrm{CH}$ shock release into vacuum are compared with the results of laser-driven experiments. The remaining part of the paper is organized as follows: Sec. II describes details of our CMD simulations and shock-release experiments; Sec. III presents theoretical and experimental results on shock strengths, hydrogen isotopes, and preheat and discusses their effects on the shock release of $\mathrm{CH}$; finally, Sec. IV summarizes all findings and concludes the paper.

\section{METHODOLOGY}

\section{A. Classical molecular-dynamics simulations}

Our CMD calculations are done with the LargeScale Atomic/Molecular Massively Parallel Simulator (LAMMPS) [49]. Considering that many-body reactive potentials are more accurate than nonreactive ones in describing interatomic interactions in hydrocarbons [50], we choose in our study the modified adaptive intermolecular reactive empirical bond order (AIREBO-M) potential, which was developed for applications to high-pressure hydrocarbons [51]. This potential has been employed to study
$\mathrm{CH}$ polystyrene under a very strong shock of $\sim 2000 \mathrm{GPa}$, and the predicted release and species separation offer a new explanation to experimental observations [39].

Our structural model of polystyrene consists of $1.35 \times 10^{6}$ atoms. It is made of 96 syndiotactic polymer chains aligning along the $z$ direction and has a thickness $z_{0}$ of about $61.7 \mathrm{~nm}$ and dimensions of $14.3 \times 15.7 \mathrm{~nm}$ in transverse directions, where the periodic boundaries condition is applied. The structural unit [see the inset of Fig. 3(c)] includes two randomly generated void regions ( $\sim 10 \mathrm{~nm}$ in size), and its average density stabilizes to $1.05 \mathrm{~g} / \mathrm{cm}^{3}$ at the ambient condition $(0 \mathrm{GPa}$, $300 \mathrm{~K})$, resembling that of real experimental samples. More details can be found in our recent work [39].

The shock is generated by using a rigid piston wall, which compresses the sample along the $+z$ direction in a fixed velocity $u_{\mathrm{p}}$ of $5-100 \mathrm{~km} / \mathrm{s}$. The piston functions by using a momentum mirror technique, which reflects any atom that hits it. This technique has been used in previous CMD simulations of materials under shock compression [52]. The simulation time step varies between 0.0125 and $0.25 \mathrm{fs}$, depending on the value of $u_{\mathrm{p}}$.

Results of the shock and the release are obtained by dividing the regions of interest into equally sized bins along the $z$ axis and running statistical analysis for atoms in each bin, which is a typical approach in CMD literature [52,53]. Specifically, densities are calculated by $\rho_{n}=\sum_{i} m_{n}^{i} / V_{n}$, where $m_{n}^{i}$ denotes the mass of atom $i$ in bin $n$, whose volume is $V_{n}$; pressure $P_{n}=\sum_{i}\left(k_{\mathrm{B}} T_{n}+\mathbf{W}_{n}^{i}\right) / V_{n}$, where $k_{\mathrm{B}}$ is the Boltzmann constant, $T_{n}=\left\langle m_{n}^{i}\left(\mathbf{v}_{n}^{i}-\mathbf{v}_{n}\right)^{2} / 2\right\rangle / 3 k_{\mathrm{B}}$ is the temperature, $\mathbf{v}$ is the velocity, and $\mathbf{W}$ is the virial stress [54]. Note that the center-of-mass velocity of each bin $\mathbf{v}_{n}$ must be removed from the velocity of each atom $\mathbf{v}_{n}^{i}$, in the calculation of temperatures.

An example of the details of computation and shock analysis is shown in Fig. 1(a) and in Fig. S1(a) of the Supplemental Material. The pressure and density profiles at any moment before shock breakout consist of three (shocked, shock front, and unshocked) stages. The average and bounds in the shocked region are taken as values and error bars, respectively, of the postshock properties [Figs. 1(c) and S1(b)] [55]. The shock velocity $u_{\mathrm{s}}$ is determined by defining the half value of the postshock pressure as the location of the shock front [horizontal dashed line in Fig. 1(a)] and linearly fitting to time [Figs. 1(b) and 1(d)], while the fitting error is used to define the error bar in $u_{\mathrm{s}}$. Note that the postshock region is highly structural, which is originated from the porous nature of our structural model, comparable sizes of the voids to that of the simulation cell, and the possibility that the system has not completely reached thermodynamic equilibrium within subpicosecond time scales during the simulation. This leads to relatively large error bars of the postshock properties. Regardless, Fig. 1(c) shows that our calculated postshock $P-u_{\mathrm{p}}$ results are accurate, while $u_{\mathrm{s}}$ are slightly higher (by up to $\sim 7 \%$ ) and postshock $\rho$ are lower (by up to $20 \%$ ), in comparison to experimental and first-principles [density functional theory molecular dynamics and path integral Monte Carlo (DFT-MD-PIMC)] Hugoniots [56]. This level of accuracy is remarkable in comparison to the errors in Hugoniots expected based on previous CMD simulations [50], which are 10-30\% at 40-80 $\mathrm{GPa}$ and larger at higher pressures. 

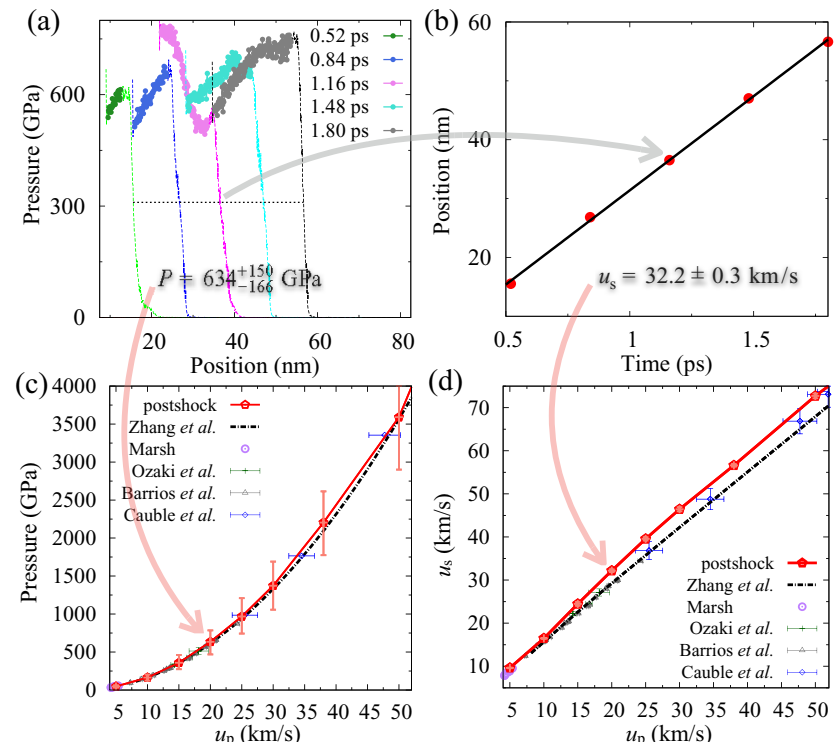

FIG. 1. (a) and (b) Schematics of the procedures of calculating the postshock properties based on CMD simulations at $u_{\mathrm{p}}=20$ $\mathrm{km} / \mathrm{s}$ and (c) and (d) the results in pressure and shock velocity at different $u_{\mathrm{p}}$ values. The colored circles in (a) denote data chosen in the shocked regime, whose averages and upper and lower bounds define the postshock properties and their uncertainties (specified in text). The dashed horizontal line in (a) denotes the location of the shock front defined by midpressure positions, which are linearly fit to time to get the shock velocity $u_{\mathrm{s}}$ in (b). In (c) and (d), the postshock $P$ and $u_{\mathrm{s}}$ based on CMD are compared with first-principles DFTMD-PIMC (Zhang et al. [30,31]) and experimental (Los Alamos Scientific Laboratory (LASL) database (Marsh [20]), Gekko laser (Ozaki et al. [22]), OMEGA laser (Barrios et al. [23]), and Nova laser (Cauble et al. [21])) Hugoniots of $\mathrm{CH}$. The initial density $\rho_{0}$ is $1.05 \mathrm{~g} / \mathrm{cm}^{3}$ in all cases.

Our calculations of the release are by continuing the piston compression at fixed velocities of $u_{\mathrm{p}}$. We have stopped the piston at various times and for selected shock strengths to understand the rarefaction-wave effects on the release. In order to study the effect of radiation preheat, we have constructed additional models for thermally expanded $\mathrm{CH}$ and compared the shock-release profiles with that of initially ambient $\mathrm{CH}$. The expanded polystyrene structures were built by scaling temperatures up to $5000 \mathrm{~K}$ and putting a motionless piston next to the left side $(z \sim 0 \mathrm{~nm})$ of the previously constructed ambient model for $\mathrm{CH}$. This allows the system to naturally expand toward the $+z$ direction. After the system expands for 5 or 10 ps (or by $\sim 20-50 \%$ in thickness), the piston velocity is reset to the desired value, and the compression starts. The shock and release profiles are then computed by following the same procedures as outlined above.

\section{B. Shock-release experiments}

Our $\mathrm{CH}$ shock-release experiments were conducted at the Omega Laser Facility at the University of Rochester's Laboratory for Laser Energetics. A planar target design was employed, which consists of a polystyrene $(\mathrm{CH})$ ablator with aluminum overcoat to prevent shinethrough and a brominated
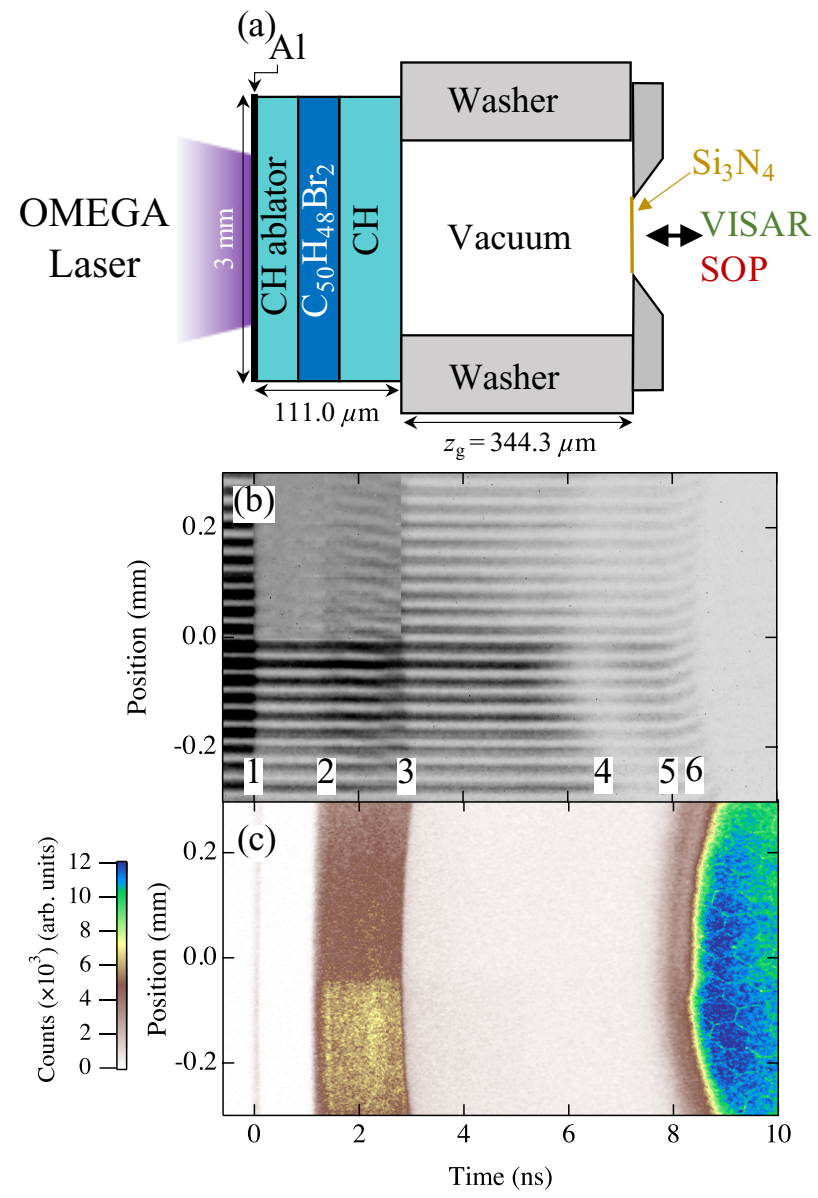

FIG. 2. (a) A schematic of the target for the $\mathrm{CH}$ shock-release experiments in this paper and representative (b) VISAR and (c) SOP images from shot 64755. In (a), the upper half of the $\mathrm{Si}_{3} \mathrm{~N}_{4}$ foil has a titanium flash coating, and some key geometric lengths of the target are labeled. In (b), the numbers 1-6 denote the times of the laser turning on, the first visibility of the shock front, the shock breakout of the $\mathrm{CH}$ sample, the reflectivity change of the $\mathrm{Si}_{3} \mathrm{~N}_{4}$ foil, the fringe shift indicating motion of the foil, and the blanking out of fringes and a sudden big increase in SOP, respectively. A ghost subtraction [57] has been applied to the upper left portion of the VISAR image in (b).

plastic layer $\left(\mathrm{C}_{50} \mathrm{H}_{48} \mathrm{Br}_{2}\right)$ to prevent preheating of the $\mathrm{CH}$ sample by $\mathrm{x}$ rays from the ablation plasma, followed by the undoped $\mathrm{CH}$ sample, as shown in Fig. 2(a). The 351-nm UV laser in a 2-ns temporally square pulse irradiated the target having a focal-spot diameter of $\sim 880 \mu \mathrm{m}$ through the use of distributed phase plates. The laser energy was adjusted to control the strength of the shock that transits the sample. When the shock exits the $\mathrm{CH}$ sample, the $\mathrm{CH}$ releases across a vacuum gap that was created using a washer with a thickness of $z_{\mathrm{g}} \sim 340 \mu \mathrm{m}$. The released materials subsequently pile up on a thin 50 -nm silicon nitride $\left(\mathrm{Si}_{3} \mathrm{~N}_{4}\right)$ foil (half coated with titanium and held by a silicon frame) and cause the foil to move. Specifics of the laser drive and the target for two shots reported in this paper are summarized in Table I.

A two-channel line-imaging velocity interferometer system for any reflector (VISAR) [57] operated with a 532-nm laser was used to measure the shock velocity in the $\mathrm{CH}$ 
TABLE I. Specifics of the 2-ns laser drive and the thickness of each layer in the target packages used in this paper. The Al overcoat is approximately $0.1 \mu \mathrm{m}$.

\begin{tabular}{lccccc}
\hline \hline Shot No. & $\begin{array}{c}\text { Laser energy } \\
(\mathrm{J})\end{array}$ & $\begin{array}{c}\mathrm{Al} / \mathrm{CH} \text { ablator } \\
(\mu \mathrm{m})\end{array}$ & $\begin{array}{c}\mathrm{C}_{50} \mathrm{H}_{48} \mathrm{Br}_{2} \text { sample } \\
(\mu \mathrm{m})\end{array}$ & $\begin{array}{c}\mathrm{CH} \text { sample } \\
(\mu \mathrm{m})\end{array}$ & $\begin{array}{c}\mathrm{Vacuum} \mathrm{gap}_{(2 \mathrm{~m})} \\
\left(\mu \mathrm{N}_{4}\right.\end{array}$ \\
\hline 64755 & 851 & 30.5 & 29.0 & 49.5 & 344.3 \\
64742 & 540 & 30.5 & 27.5 & 49.5 & 339.3 \\
\hline \hline
\end{tabular}

sample prior to its release and the velocity of the silicon nitride foil once it began accelerating. The VISAR data were supplemented by streaked optical pyrometer (SOP) $[58,59]$ data, which show the thermal emission from the shock front and the heated foil over the 590-900$\mathrm{nm}$ wavelength range. The VISAR data were analyzed using a one-dimensional (1D) fast Fourier transform algorithm, which determines the fringe position and velocities to a high precision [23,57]. Representative VISAR and SOP images from our experiments are shown in Figs. 2(b) and 2(c).

\section{RESULTS AND DISCUSSION}

\section{A. Effects of shock strength on $\mathrm{CH}$ release}

We have calculated release profiles for $\mathrm{CH}$ upon and after breakout of shocks with various strengths. Figure 3 shows the species distribution profiles from four different shocks, corresponding to $u_{\mathrm{p}}$ values of $10,15,20$, and $50 \mathrm{~km} / \mathrm{s}$, or Hugoniot pressures of $164,358,634$, and $3590 \mathrm{GPa}$, respectively. The results show hydrogen species ahead of carbon upon breakout of strong shocks $\left(u_{\mathrm{p}} \geqslant 15 \mathrm{~km} / \mathrm{s}\right)$. This is a signature of species separation that is most evident under the Hugoniot pressure of $3590 \mathrm{GPa}$, less noticeable at 358-634 GPa, and almost absent
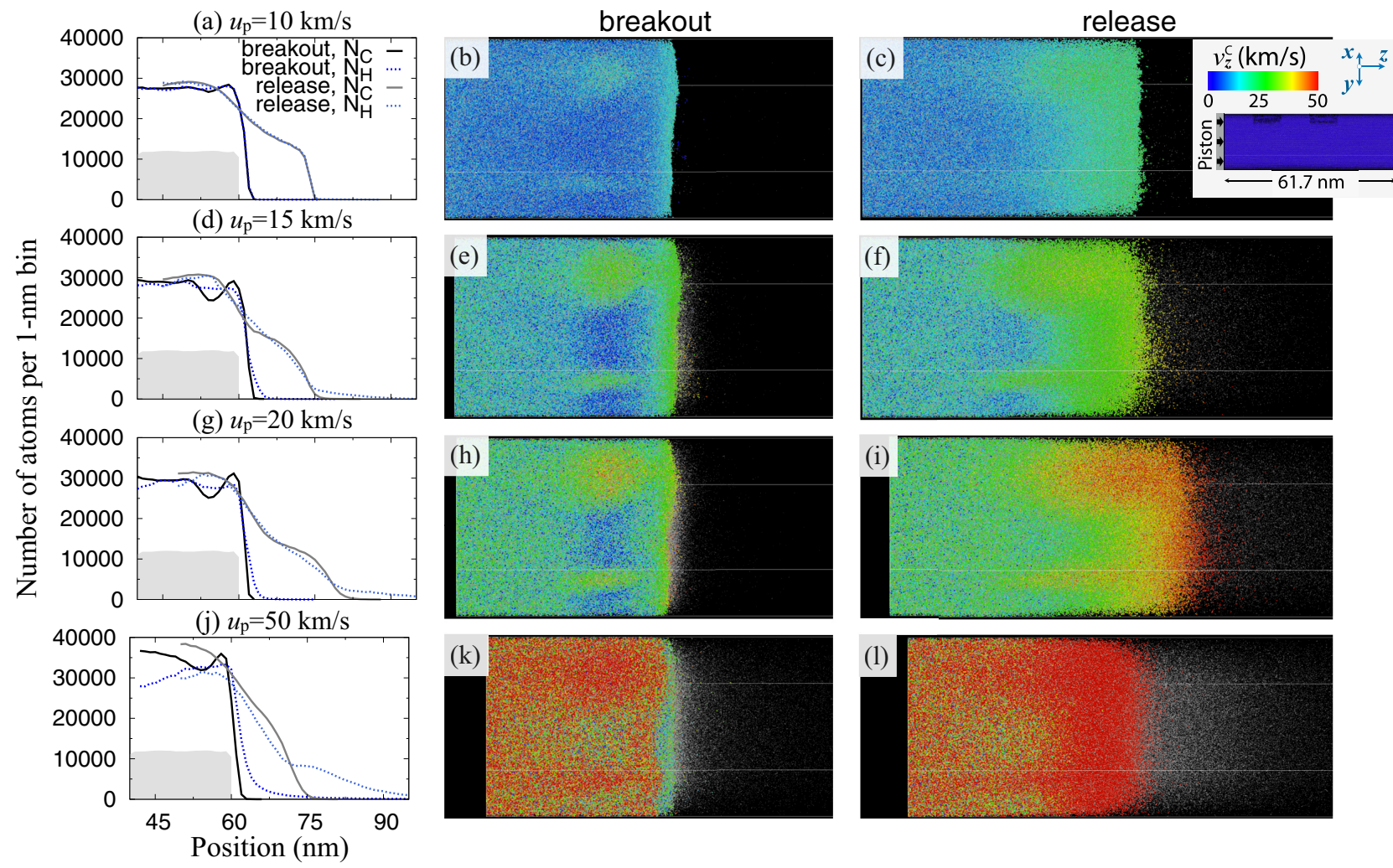

FIG. 3. Carbon and hydrogen distribution upon breakout from $\mathrm{CH}$ and during the release (at a time of $\sim 0.2 t_{0}$ after shock breakout, where $t_{0}$ is the duration of shock propagation through the sample in each case) of shocks with different strengths: (a) $-(\mathrm{c}) u_{\mathrm{p}}=10 \mathrm{~km} / \mathrm{s},(\mathrm{d})-(\mathrm{f}) u_{\mathrm{p}}=15$ $\mathrm{km} / \mathrm{s}$, (g)-(i) $u_{\mathrm{p}}=20 \mathrm{~km} / \mathrm{s}$, and (j)-(l) $u_{\mathrm{p}}=50 \mathrm{~km} / \mathrm{s}$. In the left column, gray-shaded regions denote the corresponding profiles of the initial unshocked sample. In the middle and right columns (prepared using Open Visualization Tool (OVITO) [60]), carbon atoms are color-coded according to their $z$-component velocities, hydrogen atoms are shown as white dots, and the simulation cells are viewed along an arbitrarily chosen off-diagonal direction in the $x-y$ plane (see Figs. S9-S11 in the Supplemental Material [61] for comparisons between snapshots from different views). Inset: the scale of the velocity color maps and a side view of the initial setup of the CMD simulations (same for all four simulations shown here). Note that the nonuniformity in the velocity color map in the $x$ - $y$ plane is due to local release into voids and formation of hot spots and that the voids are introduced to our structural models to resemble the plastic samples in real experiments. 
TABLE II. Key parameters for scaling and comparing simulations with experiments in this paper. $u_{\mathrm{p}}$, piston velocity; $z_{\mathrm{g}}$, thickness of the vacuum gap; $t_{\mathrm{s}}$, scaling parameter in time. All CMD simulations use $\mathrm{CH}$ samples with initial thickness $z_{0}=61.7 \mathrm{~nm}$, and $u_{\mathrm{s}}$ denotes shock velocity in CMD bounded by the postshock and first-principles Hugoniot values [see Fig. 1(d) and Table S1] or in experiment at the breakout time. Experimental $u_{\mathrm{p}}$ values were calculated from the $u_{\mathrm{s}}$ using the $\mathrm{CH}$ Hugoniot fit from Ref. [32].

\begin{tabular}{lcccc}
\hline \hline & $\begin{array}{c}u_{\mathrm{p}} \\
(\mathrm{km} / \mathrm{s})\end{array}$ & $\begin{array}{c}u_{\mathrm{s}} \\
(\mathrm{km} / \mathrm{s})\end{array}$ & $\begin{array}{c}z_{\mathrm{g}} \\
(\mathrm{nm})\end{array}$ & $\begin{array}{c}t_{\mathrm{s}} \\
(\mathrm{ps})\end{array}$ \\
\hline CMD & 10.0 & $15.9-16.5$ & 370.2 & $22.46-23.28$ \\
CMD & 15.0 & $22.5-24.5$ & 370.2 & $\begin{array}{c}15.13-16.49 \\
\text { CMD }\end{array}$ \\
CMD & 50.0 & $35.5-39.6$ & 370.2 & $9.36-10.41$ \\
& & $68.3-72.7$ & 370.2 & $5.09-5.42$ \\
Shot No. & $\begin{array}{c}u_{\mathrm{p}} \\
(\mathrm{km} / \mathrm{s})\end{array}$ & $\begin{array}{c}u_{\mathrm{s}} \\
(\mathrm{km} / \mathrm{s})\end{array}$ & $\begin{array}{c}z_{\mathrm{g}} \\
(\mu \mathrm{m})\end{array}$ & $\begin{array}{c}t_{\mathrm{s}} \\
(\mathrm{ns})\end{array}$ \\
\hline 64755 & $22.26 \pm 0.13$ & $32.17 \pm 0.16$ & 344.3 & 10.70 \\
64742 & $19.24 \pm 0.11$ & $28.21 \pm 0.15$ & 339.3 & 12.03 \\
\hline \hline
\end{tabular}

at $164 \mathrm{GPa}$. At later times during the release, larger amounts of hydrogen are found ahead of carbon, indicating growing degrees of separation of the two species.

As we have shown in Ref. [39], carbon and hydrogen species have different velocity profiles under compression of a strong shock. The broadness of the velocity profile of a species in the shocked regime is determined by the temperature following the Maxwell-Boltzmann distribution for an ideal gas. The lighter a species is, the more broadly its velocity distribution spreads. Therefore, at high velocities, we can expect a larger amount of hydrogen than carbon in $\mathrm{CH}$ shocked to above 200-400 GPa, where chemical bond dissociation occurs $[23,31,62]$. Upon shock breakout, which is governed by the conservation of momentum and energy, particles experience dramatic increase in kinetic energy that can exceed the chemical bonding energy. This further facilitates species separation in the released regime of strong shocks and explains the dependence on shock strength as shown above.

In order to fully understand the deep release process and enable comparisons among different simulations and experiments, we have calculated the velocity, density, and momentum changes with time for various shock strengths. Because of the vast differences in sizes of the sample, times of the shock-release processes, and strengths of shocks, the data would span a wide range without an appropriate scaling that can provide a coherent description of the results. Here, we set the moment of shock breakout from the sample as the origin of time $t$, chose the thickness $z_{\mathrm{g}}$ of the gap between a "witness foil" and the sample and the velocity of shock upon breakout $u_{\mathrm{s}}$, and defined the ratio between the two as the scaling factor $t_{\mathrm{s}}=z_{\mathrm{g}} / u_{\mathrm{s}}$. The values of the key parameters in our simulations and experiments are listed in Table II.

Our simulation results of $\mathrm{CH}$ release for $z_{\mathrm{g}}=6 z_{0}$ under four different values of shock strength $\left(u_{\mathrm{p}}=10-50 \mathrm{~km} / \mathrm{s}\right)$ are plotted in Fig. 4 as functions of the reduced time $t / t_{\mathrm{s}}$. They show that faster atoms arrive at the "foil" position earlier and therefore the observed velocity at the foil decreases with time.
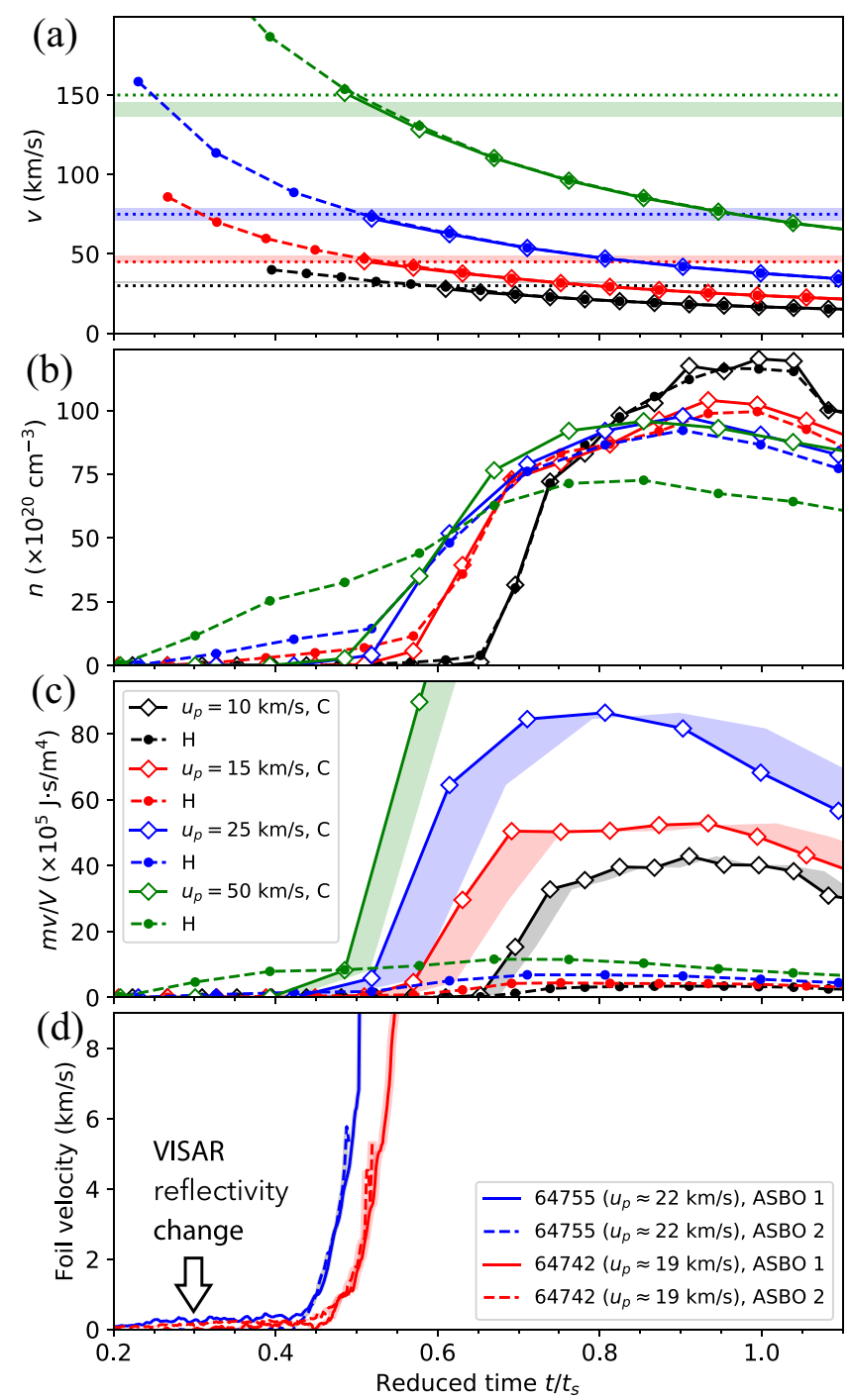

FIG. 4. (a)-(c) CMD-predicted temporal changes in species velocity (a), density (b), and momentum (c) of released $\mathrm{CH}$ at the position of a "witness foil" and (d) experimentally measured foil velocities after breakout of shocks with different strength. Time is reduced by the scaling factor $t_{\mathrm{s}}=z_{\mathrm{g}} / u_{\mathrm{s}}$, where $z_{\mathrm{g}}=370.2 \mathrm{~nm}(\mathrm{CMD})$ or $\sim 344 \mu \mathrm{m}$ (experiments) is the thickness of the gap between the foil and the sample and $u_{\mathrm{s}}$ is the shock velocity at the breakout time. In (a), dotted horizontal lines and shaded bars denote the values of $3 u_{\mathrm{p}}$ and $2 u_{\mathrm{s}}$, respectively. In (c), shaded areas denote the estimated uncertainties in time bounded by the postshock and first-principles Hugoniot values [see Fig. 1(d) and Table S1; curves with diamonds correspond to the first-principles Hugoniot values] of $u_{\mathrm{s}}$ (and thus in $\left.t_{\mathrm{s}}\right)$. In (d), shaded areas denote the estimated uncertainty in the experimental data (see Fig. 5), and the time of VISAR reflectivity change in shot 64755 is indicated with an arrow. Note that the origin of the reduced time is the moment of shock breakout. ASBO, active shock breakout.

Both the magnitude of the velocity and the rate of its decay are larger for stronger shocks, as shown in Fig. 4(a). The fastest hydrogen atoms arrive at the foil first, and then both hydrogen and carbon atoms having slower velocities arrive at later times. 
Temporal changes in density of the released species that arrive at the "foil" are more abrupt than in velocity, as shown in Fig. 4(b). The densities of both species first increase and then decrease, corresponding to velocity distributions that are Gaussian in the shocked regime and modulated by breakout of the shock. This modulation results in steepened density curves for early-arrived, fast species and flattened ones for late-arrived, slow species. For strong shocks $\left(u_{\mathrm{p}} \geqslant 15 \mathrm{~km} / \mathrm{s}\right)$, Fig. 4(b) shows that the hydrogen density at the foil location is initially higher and then lower than that of carbon, more so for stronger shocks due to the more significant separation of the species. The maximum in density of hydrogen $n_{\mathrm{H}}$ is lower than that of carbon $n_{\mathrm{C}}$, which compensates the higher $n_{\mathrm{H}}$ than $n_{\mathrm{C}}$ at earlier time because the amounts of $\mathrm{C}$ and $\mathrm{H}$ are equal in the initial sample. The density maximum appears earlier in reduced time when the shock is stronger, except for the case of $u_{\mathrm{p}}=10 \mathrm{~km} / \mathrm{s}$, in which the maximum occurs between those of $u_{\mathrm{p}}=15$ and $25 \mathrm{~km} / \mathrm{s}$. Moreover, the hydrogen and carbon density profiles are very similar for the case of weak shock release $\left(u_{\mathrm{p}}=10 \mathrm{~km} / \mathrm{s}\right)$, which implies that chemical bonding is playing more important roles at those low pressures.

Figure 4(c) shows momentum per unit volume of the species during the release, which is a product of velocity and density, at the location of the foil. Overall, the impulse of the release slowly ramps up first with the increasing amount of hydrogen, then dramatically increases upon the arrival of carbon, and later gradually decreases as velocity and density decrease. Because of the monotonic changes in velocity and the much lower mass of hydrogen than carbon nuclei, the feature of the momentum curves is dominated by that of the density curves of carbon [as shown in Fig. 4(b)], while hydrogen contributions are relatively weak and best visible in the plot only for the strongest shock. Nevertheless, the weak contribution from hydrogen begins at an earlier time than carbon and more so for stronger shocks, which is associated with the species separation upon breakout of strong shocks. The carbon momentum curves clearly differentiate between shocks of different strength, even after taking into account the uncertainties in reduced time (shaded regions). The momentum jump from the arrival of the carbon species occurs earlier and is larger in value for stronger shocks. We also see that the hydrogen atoms streaming ahead of the carbon have a larger contribution to the early increase in momentum for stronger shocks. The onset of momentum jump in $t / t_{\mathrm{s}}$ ranges from $0.61-0.65$ for $u_{\mathrm{p}}=10 \mathrm{~km} / \mathrm{s}$ to $0.41-0.50$ for $u_{\mathrm{p}}=50 \mathrm{~km} / \mathrm{s}$. The values are around 0.5 because the maximum carbon velocity is approximately $2 u_{\mathrm{s}}$, or around $3 u_{\mathrm{p}}$ (similar to previous findings based on hydrodynamic models [63]), as shown with colored horizontal bands or dotted lines in Fig. 4(a).

We also performed calculations using a two-times-larger cell and compared the results for different vacuum gap thicknesses $z_{\mathrm{g}}$, and we found that the overall pictures remain the same (see the Supplemental Material [61]). We thus use the approach outlined here to compare the simulations and experiments that have different length and time scales.

\section{B. Comparing CMD simulations with experiments}

We see an abrupt momentum jump in the simulations as the carbon species reaches the location of the witness foil.

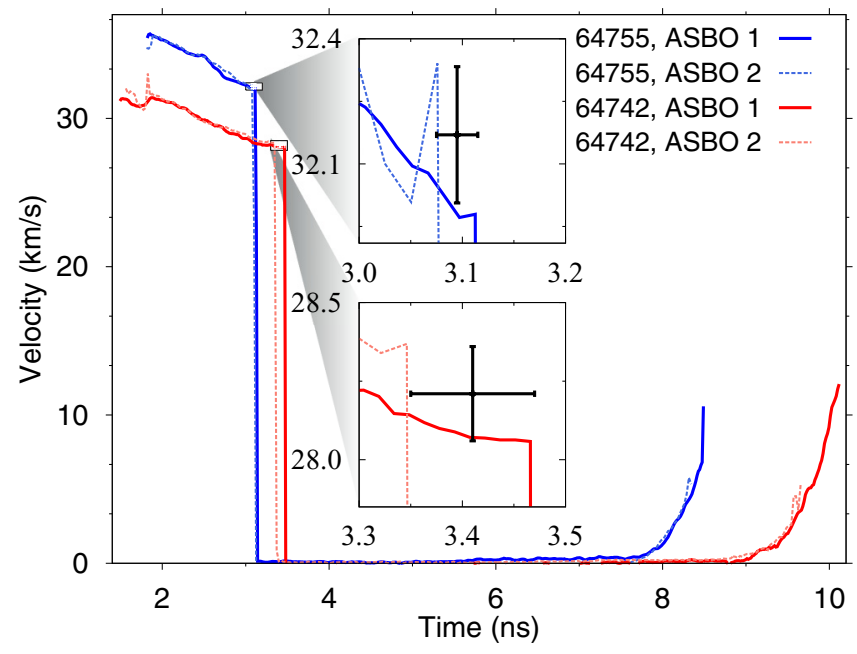

FIG. 5. The VISAR-measured shock and $\mathrm{Si}_{3} \mathrm{~N}_{4}$ foil velocities in two shots. Solid and dashed curves denote results from two different cameras (ASBO 1 and 2). The insets are zoomed-in views of the velocity profiles at breakout. The black crosses in the insets and their error bars denote the shock velocities upon breakout and their uncertainties, respectively, estimated by the average and differences between the two VISAR records. These uncertainties are propagated to estimate the uncertainty in the reduced time plot shown with shaded areas in Fig. 4(d).

Accordingly, we can expect the velocity of the $\mathrm{Si}_{3} \mathrm{~N}_{4}$ foil to increase in our shock-release experiments because of momentum transfer to the foil by piling up of the species (see the Supplemental Material [61]). Prior to the velocity jump, early arrival of high-speed low-mass hydrogen species does not bring enough momentum to move the foil. Instead, they could penetrate into the foil or pile up on its surface and induce observable changes in its optical properties (see the Supplemental Material [61]). Such optical signatures are expected to be experimentally detectable only under strong shocks with significant enough species separation. These theoretical predictions based on our CMD simulations are compared with our experimental results in the following.

Figure 5 shows the velocity profiles from our VISAR measurements for two shots. For each shot, we measure the shock velocity in the $\mathrm{CH}$ sample and the velocity of the $\mathrm{Si}_{3} \mathrm{~N}_{4}$ foil later in time. The shock breaks out of the $\mathrm{CH}$ sample at approximately 3.1 and $3.4 \mathrm{~ns}$ for the stronger and weaker shock shots, respectively. Note that the VISAR does not "see" all the way through the $\mathrm{C}_{50} \mathrm{H}_{48} \mathrm{Br}_{2}$ layer, which is not fully transparent [64]. For the strong shock shot (blue lines), $u_{\mathrm{s}}$ decreases from $35.2 \pm 0.4$ to $32.2 \pm 0.2 \mathrm{~km} / \mathrm{s}$; for the weaker shock shot (red lines), it drops from $31.6 \pm 0.4$ to $28.2 \pm$ $0.2 \mathrm{~km} / \mathrm{s}$. The two cases are comparable to our CMD simulations with $u_{\mathrm{p}}=25 \mathrm{~km} / \mathrm{s}$ (Hugoniot pressure $\sim 967 \mathrm{GPa}$ ) and $20 \mathrm{~km} / \mathrm{s}$ (Hugoniot pressure $\sim 634 \mathrm{GPa}$ ), while the values of $u_{\mathrm{s}}$ at the time of shock breakout correspond to $u_{\mathrm{p}} \approx 22$ and $19 \mathrm{~km} / \mathrm{s}$ based on the shock Hugoniot of $\mathrm{CH}$ (see Table II), respectively.

After shock breakout [labeled as time 3 in Fig. 2(b)], our interferometry and pyrometry diagnostics detect the effect of the $\mathrm{CH}$ release on the $\mathrm{Si}_{3} \mathrm{~N}_{4}$ foil. Interestingly, we observed 
changes in reflectivity of the sample package [labeled as time 4 in Fig. 2(b)] prior to any shifts and eventual blanking out [labeled as times 5 and 6, respectively, in Fig. 2(b)] of the VISAR fringes. This indicates changes in optical properties of the foil before it is physically moved by the released $\mathrm{CH}$. We interpret such optical changes as a result of the high-speed [>120 km/s; see Fig. 4(a)] hydrogen streaming ahead of the bulk $\mathrm{CH}$ that impacts the $\mathrm{Si}_{3} \mathrm{~N}_{4}$ foil and changes its optical absorption and the measured reflectivity. We note that the time of reflectivity change $(\sim 6.3 \mathrm{~ns}$ in the VISAR record, or $\sim 3.2 \mathrm{~ns}$ after shock breakout) corresponds to a value of 0.3 in scaled time $t / t_{\mathrm{s}}$ [65], by which our CMD simulations predict that the amount of hydrogen that arrives at the location of the foil has increased to over $10^{20} \mathrm{~cm}^{-3}$, as shown in Fig. 4(b). We also note that similar reflectivity changes have been observed using VISAR in all our glow-discharge-polymer and $\mathrm{CH}$ experiments when the plastics are shocked to above $550 \mathrm{GPa}$, but not in release experiments of pure diamond at $1000 \mathrm{GPa}$ or beryllium at $150 \mathrm{GPa}$ [66]. These observations are consistent with species separation, which is expected in the release of $\mathrm{CH}$ materials under strong shocks but does not occur for elemental solids, and provide quantitative support for our computational findings.

After the $\mathrm{CH}$ releases across the vacuum gap, our VISAR streak images show shifts of the fringes that correspond to motion of the $\mathrm{Si}_{3} \mathrm{~N}_{4}$ foil [labeled as time 5 in Fig. 2(b)]. The foil velocities are plotted in Fig. 5, where the foil begins moving at $\sim 7.8$ and $\sim 9 \mathrm{~ns}$ for the stronger shock (shot 64755 ) and weaker shock (shot 64742), respectively. The foil velocity profiles are replotted with respect to the reduced time $t / t_{\mathrm{s}}$ in Fig. 4(d). It shows that the foil velocity ramps up abruptly for both shots, and the onset in reduced time $t / t_{\mathrm{S}}$ is earlier for the strong shock (shot 64755) than for the weak one (shot 64742). This is in agreement with the trend predicted by our CMD simulations [Fig. 4(c)].

We note that the experimental onsets of the foil acceleration happen at $t / t_{\mathrm{S}}$ of $0.45-0.5$ in shot 64742 and at $0.42-0.46$ in shot 64755 , which seems slightly earlier than the theoretically predicted momentum jumps [Figs. 4(c) and 4(d)]. In addition, the shorter duration of the laser drive $(\sim 2.0 \mathrm{~ns})$ than the shock transit time ( $\sim 3.1-3.4 \mathrm{~ns})$ indicates that laser ablation has stopped before shock breakout, which could produce rarefaction waves that affect the release; in contrast, the piston drive in the simulations is constant. In order to understand these differences and their effects, we performed additional simulations by considering different vacuum gap thickness, sample sizes, and simulation setup. Considering the uncertainties in $t_{\mathrm{s}}$ predicted by CMD, we chose a broader range in shock strength $u_{\mathrm{p}}=20-38 \mathrm{~km} / \mathrm{s}$ for these studies in order to obtain a clearer picture in physics.

By comparing several simulation results for $u_{\mathrm{p}}=38 \mathrm{~km} / \mathrm{s}$ and different values of vacuum gap thickness, we found momentum profiles for the early-released hydrogen and the onset of carbon momentum jump that are overall the same, whether or not the piston is stopped while the shock is still transiting in the sample (see Supplemental Material [61]). Considering the major uncertainties (shaded areas in Fig. 4) that originate from the errors in the CMD-predicted $u_{\mathrm{s}}$, it remains difficult to accurately differentiate the results between $u_{\mathrm{p}}=20$ and 25 $\mathrm{km} / \mathrm{s}$ (or Hugoniot pressures 634 and $960 \mathrm{GPa}$ ) by our CMD
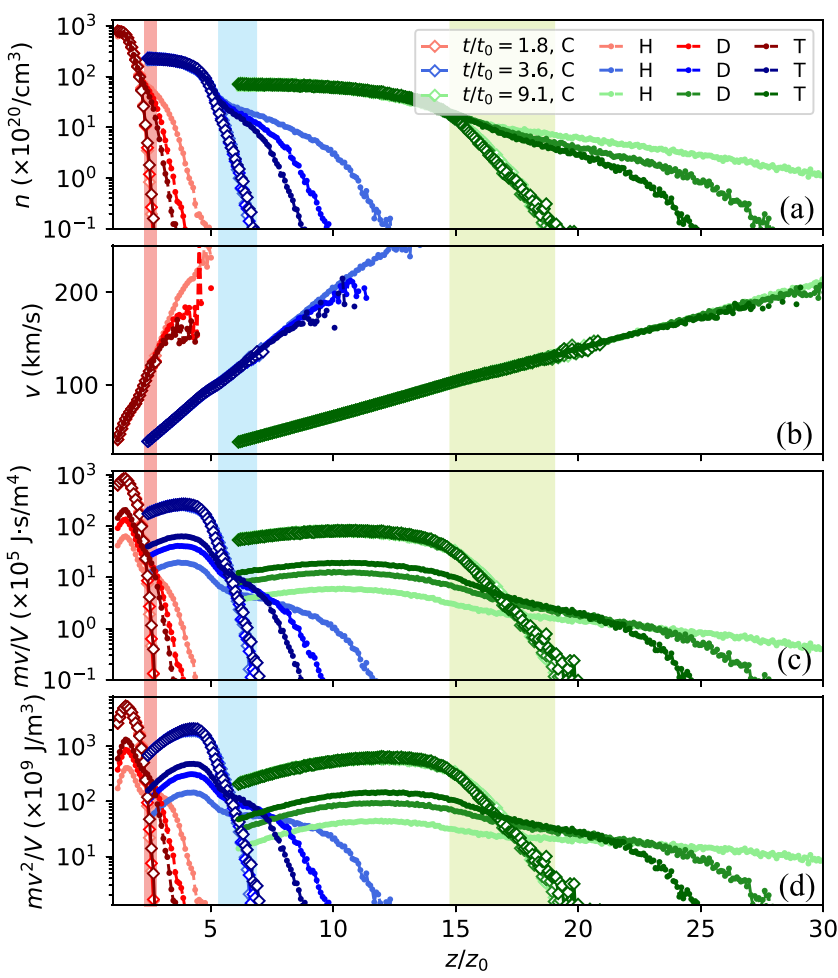

FIG. 6. Spatial distribution in (a) density, (b) velocity, (c) momentum, and (d) kinetic energy of species in shock-released polystyrene with different hydrogen isotopes (with increasing color darkness: $\mathrm{CH}, \mathrm{CD}$, and $\mathrm{CT}$ ) at different times (red, blue, or green) during the release. Shaded areas (vertical bars in light colors) denote region II (carbon and hydrogen separate), which divides region I (carbon and hydrogen coexist) from region III (hydrogen streams ahead of carbon). Time starts at the moment the piston begins to compress the sample. Thickness of initial sample $z_{0}=61.7 \mathrm{~nm}$, duration of shock propagation through the sample $t_{0}=1.1 \mathrm{ps}$, and piston velocity $u_{\mathrm{p}}=38 \mathrm{~km} / \mathrm{s}$.

approach. Nevertheless, our CMD results in a broader range of shock strength clearly show a decreasing trend in the onset of momentum jump with increasing shock strength, which is in agreement with the shock strength dependence of foil velocities measured by our release experiments [Figs. 4(c) and 4(d)]. Future simulations with improved accuracy and experiments considering broader ranges of shock strength could be helpful in further understanding our present experimental findings and theoretical predictions.

\section{Effects of hydrogen isotopes}

HED and ICF experiments often involve deuterium (D) and tritium (T), in addition to hydrogen $(\mathrm{H})$. Therefore it is interesting to study the isotopic effect on species separation and the physics of shock release. We have carried out simulations of carbon-deuterium (CD) and carbon-tritium (CT) polystyrene using an initial structure, force fields, and computational settings that are the same as that for $\mathrm{CH}$ polystyrene except for the mass of the hydrogen isotopes. The results in density, velocity, momentum, and kinetic energy during the release (in red, blue, or green) are shown in Fig. 6. 
Figure 6 shows, between different simulations, that the distribution profiles are indistinguishable for carbon but clearly different for hydrogen. The number density $n$ of the released species all monotonically decrease with time $t$ and with distance $z$ from the original sample position. However, clear differences can be observed between the species. At each time, the simulation results can be divided into three regions: Region I (named the " $\mathrm{C}-\mathrm{H}$ coexisting region") is within certain distances from the piston, where the number densities of carbon and hydrogen are similar to each other and both slowly decrease with distance; region II (named the "speciesseparation region") is ahead of region I but behind the release front of carbon $\left(n \approx 10^{19} \mathrm{~cm}^{-3}\right.$ ), where the density of carbon drops at a higher rate than that of hydrogen and their differences in $n$ grow to over two orders of magnitude (OOMs) at the carbon release front; and region III (named the "hydrogenstreaming region") is between the release fronts of carbon and hydrogen, where the densities of lighter hydrogen isotopes have higher values and decrease more slowly. Overall, lighter hydrogen isotopes can stream farther ahead of carbon, which is more clearly shown at later times during the release.

Over time, all three regions expand, which results in spatially enlarged differences in density among different species. However, the overall velocity profiles for different species remain similar, particularly in regions I and II, and they grow into a linear relationship with position as the release proceeds, which indicates a nearly free motion of the high-speed species that form the release. Some minor differences between velocities of species are observed at the release fronts. Generally, a species moves faster if it is lighter and closer to its release front, and the velocity maximum of the species is inversely correlated with the atomic weight.

The three-region patterns are also carried over to the momentum and kinetic energy profiles in Figs. 6(c) and 6(d). In region I, heavier species have larger momentum and kinetic energy that are proportional to the atomic weight. In addition, all of the momentum and kinetic energy curves have a convex shape with a peak, which flattens as region I expands with time. The momentum and kinetic energy in region II are characterized by monotonic decreases, which are abrupt for carbon but relatively smooth for hydrogen species. At the carbon release front, the momentum and energy of carbon drop by three to four OOMs relative to the corresponding peak values in region I, while those of hydrogen drop by one OOM. Such one-to-two-OOM-higher momentum than carbon at the end of region II enables hydrogen to take one-to-two-OOM-higher energy (and mass) to farther distances in region III than what would otherwise be expected assuming no separation from carbon. This could result in compression degradation as well as large discrepancies between hydrodynamic simulations and experiments when using CH-based shells or layers in ICF and HED targets, in particular, if they neighbor materials that have low resistance to the flow of high-speed hydrogen.

Furthermore, in region III of the release, we observe dramatic differences between hydrogen isotopes. Lighter hydrogen species have lower momentum and energy $(\mathrm{T}>\mathrm{D}>$ $\mathrm{H}$ ) in region I, and these quantities decrease more slowly in region II. As a result, the momentum and energy of the three first become comparable at the carbon release front, and then lighter hydrogen isotopes have higher momentum and energy
$(\mathrm{H}>\mathrm{D}>\mathrm{T})$ in region III. At the release front of T, the momenta of $\mathrm{H}$ and $\mathrm{D}$ are higher than that of $\mathrm{T}$ by about one OOM, which allows $\mathrm{H}$ and $\mathrm{D}$ to take an OOM-higher energy and mass to farther distances. Similarly, at the release front of D, the momentum of $\mathrm{H}$ remains high, which allows $\mathrm{H}$ to take an OOM-higher energy and mass to even farther distances. Such differences in streaming rate of different hydrogen isotopes could reduce the compression capability of the DT ice and further affect the efficiency of the thermal nuclear reaction and ignition in the center of ICF capsules.

\section{Effects of radiation preheat}

While species separation and hydrogen streaming offer good explanations for both velocities and scale lengths of the low-density plasmas released from $\mathrm{CH}$ [39] as measured in the optical interferometry experiment [36], the CMD simulations have not considered radiation preheat that has likely occurred in this experiment and can affect the release [36-38]. In order to clarify the role of radiation, we performed additional CMD simulations of $\mathrm{CH}$ shock release using preheated samples. Following the approaches outlined in Sec. II, we constructed thermally expanded $\mathrm{CH}$ models by starting from the ambient $\mathrm{CH}$ structure, subjecting it to a high temperature of $5000 \mathrm{~K}$ $(\sim 0.42 \mathrm{eV} /$ atom in thermal energy, similar to that estimated for the experiments [36,38]), and allowing one side to expand for 5 or $10 \mathrm{ps,} \mathrm{before} \mathrm{launching} \mathrm{the} \mathrm{piston} \mathrm{and} \mathrm{compressing}$ from the other side at a speed of $38 \mathrm{~km} / \mathrm{s}$. The resultant expansion is $20-50 \%$ (or on the order of $10 \mathrm{~nm}$, depending on the initial thickness of the sample), which allows us to quantify the dependence of the release on sample changes in a reasonably broad range without significantly affecting the overall density of the bulk $\mathrm{CH}$ sample. Moreover, we have considered two different sizes of the sample with thicknesses $z_{0}=61.7$ and $123.4 \mathrm{~nm}$ in order to study the finite-size effect on the release from preheated samples.

The species density distribution profiles at different times during shock and release are shown in Fig. 7, in comparison to that of the uncompressed samples (gray- or salmon-shaded regions). In the initially ambient cases, the duration $t_{0}$ of shock propagation through the sample is 1.1 and $2.2 \mathrm{ps}$ for the thin and thick samples, respectively, and signs of separation between hydrogen and carbon are clear upon shock breakout [black curves in Figs. 7(a) and 7(d)] and enlarged during the release (blue curves), consistent with the results shown in Sec. III A. In the preheated cases, thermal expansion delays the shock breakout (onset of release) and the onset of species separation, especially for the cases of smaller samples and longer periods of preheating. We have found that the spatial range of species separation during the release is sensitive to both the degree of preheating and the thickness of the sample, as shown by the various widths of the blueshaded areas in Fig. 7. Samples preheated for 5 ps [Figs. 7(b) and $7(\mathrm{e})]$ have broader separation ranges (i.e., the width of shaded areas in light blue) than the ambient cases, while those preheated for $10 \mathrm{ps}$ [Figs. 7(c) and 7(f)] have narrower separation ranges, at the moment of $t: t_{0}=2.0: 1.1$ (for the thin sample) or, equivalently, $4.0: 2.2$ (for the thick sample). The latter (i.e., narrower separation ranges when heated for $10 \mathrm{ps}$ ) is understandable as the shock front has just exited the 

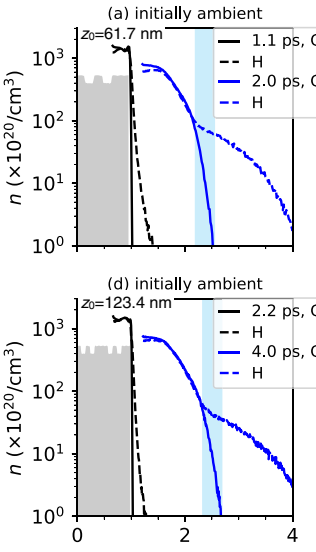
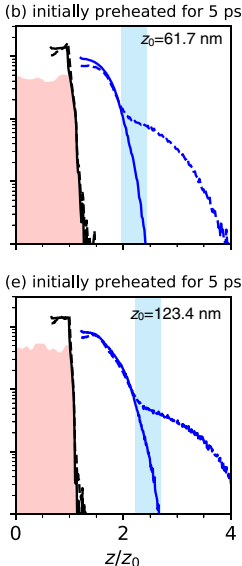

(e) initially preheated for 5 ps

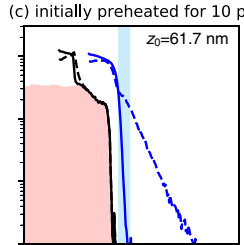

(f) initially preheated for $10 \mathrm{ps}$

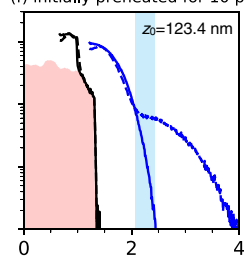

FIG. 7. Species density distributions upon or before shock breakout (black curves) or during the release (blue curves) from initially ambient [(a) and (d)] or initially preheated (thermally expanded) [(b), (c), (e), and (f)] polystyrene samples in comparison to those right before the compression starts (gray- or salmon-colored areas, $\mathrm{C}$ and $\mathrm{H}$ are not distinguishable). The initial sample at ambient conditions has a thickness $z_{0}$ of $61.7 \mathrm{~nm}$ [(a)-(c)] or $121.3 \mathrm{~nm} \mathrm{[(d)-(f)]} \mathrm{before}$ being compressed by the piston. In the preheated cases, the ambient structures are subjected to $5000 \mathrm{~K}$ and allowed to expand in the $+z$ direction for 5 ps [(b) and (e)] or 10 ps [(c) and (f)]. Solid and dashed curves correspond to carbon and hydrogen, respectively. Shaded areas in light blue denote the approximate ranges of species separation (similar to the "region II" defined in Sec. IIIC) in these cases. Time starts at the moment the piston begins to compress the sample. Piston velocity $u_{\mathrm{p}}$ is $38 \mathrm{~km} / \mathrm{s}$.

sample and the species separation has just begun, while the broader separation range in the former case (i.e., when heated for $5 \mathrm{ps}$ ) indicates that the species released from initially preexpanded samples have higher velocities than those from initially ambient $\mathrm{CH}$. Moreover, the broadening in separation ranges and enhancement in velocities of the released species by preheating are more significant when the initial sample is thicker.

Figure 8 compares the density and velocity distributions of the release at later times. We focus on the low-density regions near the front of the release, which are relevant to recent experiments [36] (our results in a broader range of densities are included in the Supplemental Material [61]). Our results, especially those on the effect of preheating, show a striking dependence on cell size. The released species from preheated samples (dark- and intermediate-colored curves) first fall behind and then gradually catch up and surpass those from unpreheated ones (light-colored curves). The differences between initially preheated and unpreheated simulations in the density of the species released from the thicker sample are initially smaller and then larger than the thinner sample [Figs. 8(a) and 8(c)]. This is because the species released from the preheated thicker sample have higher velocities, more so when the initial sample was preheated for a longer time [Figs. 8(b) and 8(d)]. The velocity enhancement due to preheating mainly affects hydrogen but also carbon, provided the sample is large enough. According to our simulations with the larger cell, the velocity increases by up to $\sim 45 \mathrm{~km} / \mathrm{s}$ for hydrogen and $\sim 10-15 \mathrm{~km} / \mathrm{s}$ (larger at lower densities) for carbon when the sample is preheated for up to $10 \mathrm{ps}$. In comparison, our simulations of the smaller cell show that the velocities of hydrogen increase (by up to $\sim 27 \mathrm{~km} / \mathrm{s}$ ) while those of carbon do not show a clear trend of increasing when the sample is preheated.

The velocities of species predicted by our CMD simulations are compared with the experimentally measured plasma velocities [36] in Figs. 8(b) and 8(d). Note that the experimental data are for the plasma electron density $n_{\mathrm{e}}$ and this can be translated into the species density by $n=n_{\mathrm{e}} /\langle Z\rangle$ via the average ionization $\langle Z\rangle$, which is bounded between 0.5 and 1 at the temperature and density conditions being considered [39]. Therefore $n_{\mathrm{e}}$ values of $10^{19}$ and $10^{20} \mathrm{~cm}^{-3}$ correspond to $n=(1-2) \times 10^{19}$ and $(1-2) \times 10^{20} \mathrm{~cm}^{-3}$, respectively. When $\mathrm{CH}$ is not preheated, we have shown that the velocities and scale lengths [67] of the low-density plasmas generated by the streamed hydrogen both match the experimental values [gray circles and black squares in Figs. 8(b) and 8(d)]. In comparison, CMD-predicted carbon velocities are much lower [gray diamonds in Figs. 8(b) and 8(d)], and scale length changes are much smaller. However, these carbon results are similar in scale lengths while lower in velocities (by approximately $25 \mathrm{~km} / \mathrm{s}$ ) than predictions by hydrodynamic simulations that did not consider radiation preheating of the sample [gray squares in Figs. 8(b) and 8(d)].

By considering improved radiation-transport models and assuming radiation-preheat-induced thermal expansion by a few micrometers (about 10\% of the thickness of the sample) at the shock-release side of $\mathrm{CH}$, follow-up hydrodynamic simulations have been reported to be able to reproduce the experimental velocities and scale lengths of the low-density plasmas [36-38]. In our CMD simulations, the $\mathrm{CH}$ sample expands by approximately $20 \%$ if preheated for 5 ps or $50 \%$ if preheated for $10 \mathrm{ps}$ (Fig. 7). Meanwhile, as the sample's initial thickness $z_{0}$ increases from 61.7 to $123.4 \mathrm{~nm}$, the carbon velocities show minimal increases due to preheating, while the carbon scale length increases by $\sim 0.14 z_{0}$ at $t: t_{0}=$ $12.0: 2.2$ or, equivalently, $6.0: 1.1$ [green curves in Figs. 8(c) and $8(\mathrm{~d})$ ]. Therefore it is possible, if extrapolating our CMD results to the experimental sample size $\left(z_{0} \approx 37 \mu \mathrm{m}\right)$ with $10 \%$ preexpansion, that the carbon velocities and scale lengths would both become comparable to the experimental values. This would then explain the results from new hydrodynamic simulations [36-38], although ideally it still needs to be tested by simulating samples up to micrometer sizes (or of the order of $10^{9}$ atoms), which is beyond the current computational capability using CMD.

Nevertheless, our CMD results suggest that such extrapolation of carbon velocities and scale lengths must be associated with hydrogen plasmas with much higher velocities [68], which is completely unexpected by hydrodynamics. As we have discussed in Sec. III C, the differences are due to species separation and hydrogen streaming, which can dramatically change the pictures given by single-fluid hydrodynamic simulations about mass, momentum, and energy distributions in the release. By taking into account such microscopic release physics in the atomic level, we expect the degree of preexpansion of $\mathrm{CH}$, if it truly occurred, to be no more than a few percent (much less than $1 \mu \mathrm{m}$ ) in order to explain the experimentally observed velocities and scale lengths of the 

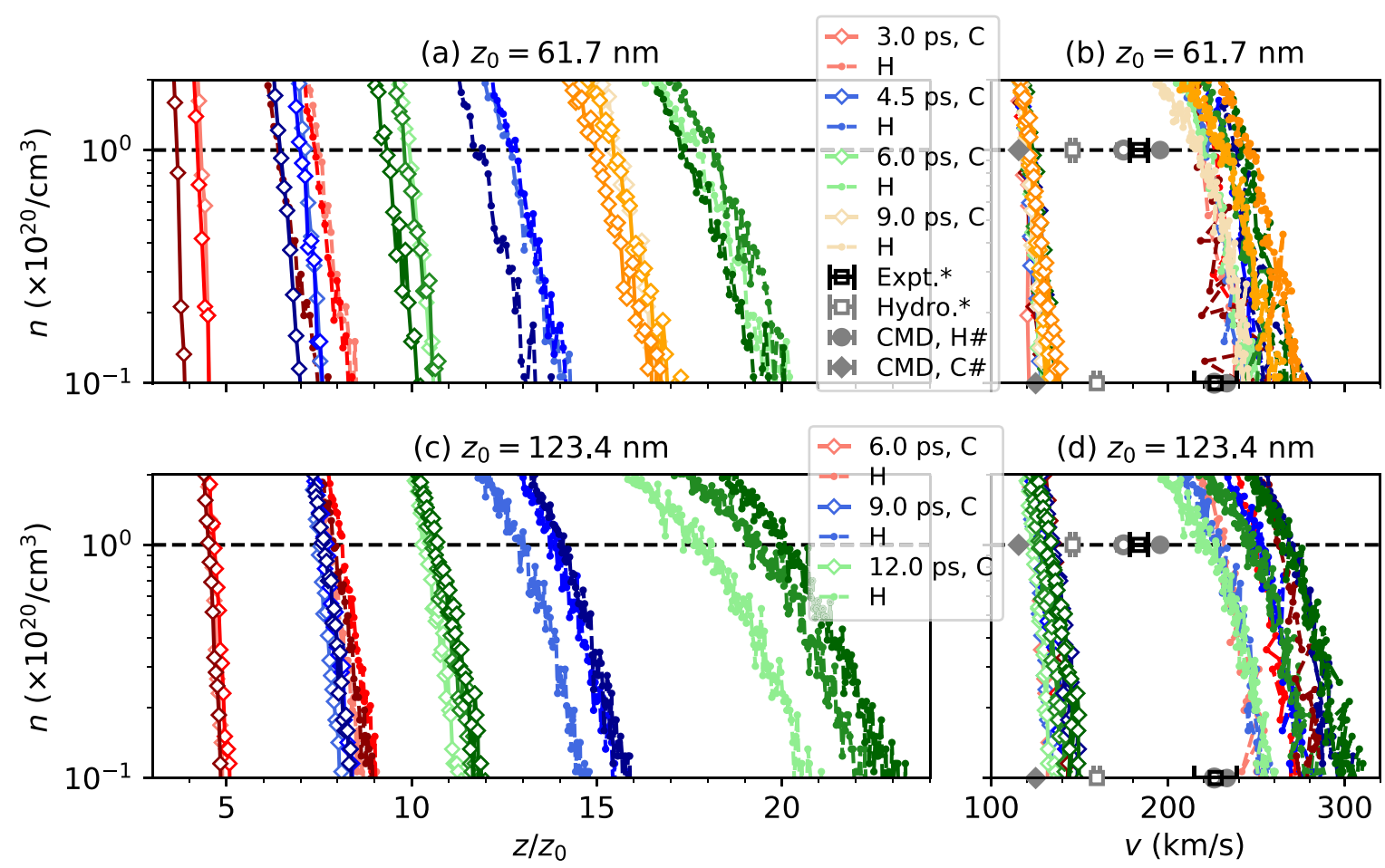

FIG. 8. (a) and (c) Spatial density profiles and (b) and (d) velocity-density relations of carbon and hydrogen species released from initially ambient (light colors) or preheated (for 5 or $10 \mathrm{ps,} \mathrm{shown} \mathrm{with} \mathrm{intermediate} \mathrm{or} \mathrm{darker} \mathrm{colors,} \mathrm{respectively)} \mathrm{CH}$ with different initial thicknesses $z_{0}$. Results for the thinner sample $(61.7 \mathrm{~nm})$ at time $t$ are shown in the same color as those for the thicker sample (123.4 $\left.\mathrm{nm}\right)$ at time $2 t$, which are equivalent for the initially unpreheated samples under linear scaling. The time origin is defined as the moment the piston begins to compress the sample. In (a), hydrogen profiles at 9.0 ps are beyond the range of the plot and not shown for clarity. In (b) and (d), plasma velocities from experiments and single-fluid hydrodynamic (Hydro.) simulations (without considering radiation preheating of the sample) from Ref. [36] (marked with "*” in the legend) and those based on CMD simulations of initially ambient CH from Ref. [39] (marked with "\#” in the legend; filled and empty circles are for hydrogen with $\langle Z\rangle=1$ and 0.5 , respectively) are shown for comparison. Scale lengths can be easily estimated from the differences in coordinates at two densities via $s=\left[z\left(n=10^{19}\right) / z_{0}-z\left(n=10^{20}\right) / z_{0}\right] / 2.3 \times z_{0}$. The velocities of the released species increase due to preheat, while the scale lengths remain similar. Another version of the figure plotted in a broader range of densities is shown in the Supplemental Material (Fig. S8) [61].

low-density plasmas [36,38]. This provides a useful prediction that is testable by future experiments.

\section{CONCLUSIONS}

To summarize, we have combined large-scale nonequilibrium CMD simulations with laser-drive experiments to study the shock release of $\mathrm{CH}$ polystyrene at shock strengths spanning from 50 to $13500 \mathrm{GPa}$. Our shock results show overall remarkable consistency (within $0-20 \%$ in comparison to deviations expected from previous simulations of $10-30 \%$ at pressures below $100 \mathrm{GPa}$ and larger at higher pressures) with experiments and first-principles Hugoniots across the entire pressure range considered.

Our experiments and simulations (scaled using the vacuum gap thickness and shock velocity at breakout) examine shocked $\mathrm{CH}$ releasing across a vacuum gap and piling up on a thin "witness foil." Our simulations of the release of shocked $\mathrm{CH}$ show separation of the carbon and hydrogen species that is more evident for stronger shocks but is almost absent when the $\mathrm{CH}$ is shocked to below $160 \mathrm{GPa}$. At the foil position, we find that the momentum of the released material slowly increases with increasing hydrogen density and then abruptly increases upon arrival of carbon species. These findings are consistent with our laser-drive experiments, which show VISAR reflectivity changes (interpreted as separated hydrogen affecting the optical properties of the $\mathrm{Si}_{3} \mathrm{~N}_{4}$ foil) before movement of the foil. By characterizing the release with a three-region pattern-region I with coexisting species, region II with separation of different species, and region III with streaming of light species-we found that lighter species or hydrogen isotopes can carry higher energy and mass than the heavier hydrogen or carbon species by one to two orders of magnitude and take them to farther distances.

Moreover, we have studied the effect of radiation preheat on shock release and found that the spatial range of species separation during the release is sensitive to both the degree of preheating and the thickness of the sample. Our CMD results suggest that samples in scales of tens of micrometers and with $10 \%$ preexpansion could have carbon velocities and scale lengths that are comparable to the experimentally measured plasma values. This could explain the different results between hydrodynamic simulations with and without proper radiation-transport models. In contrast to the hydrodynamic predictions, our CMD results suggest that such high carbon velocities must be associated with 
species-separated hydrogen plasmas with much higher velocities, and if preexpansion of $\mathrm{CH}$ truly occurred in the experiment of Haberberger et al. [36], we expect it to be far less than $1 \mu \mathrm{m}$.

This work represents a preliminary examination toward shock release in real targets of ICF and HED experiments. Our computationally and experimentally evidenced species separation and streaming of lighter species could result in compression degradation as well as large discrepancies between hydrodynamic simulations and experiments when using $\mathrm{CH}$ layers in ICF and HED targets, in particular, if they neighbor materials that have low resistance to the flow of highspeed hydrogen. The corresponding differences in streaming rate of different hydrogen isotopes could also reduce the compression capability of the DT ice and further affect the efficiency of thermal nuclear reaction and ignition in the center of ICF capsules. The microscopic physics of species separation shall be considered, and its potential effects shall be clarified $[10,14]$, in the design, interpretation, and analysis of future HED and ICF experiments. We call for future shock-release experiments with multiple species or isotopes and in broad range of shock strengths to test our findings.

\section{ACKNOWLEDGMENTS}

The authors thank J. Carroll-Nellenback for computing support and W. Unites for target fabrication. This material is based upon work supported by the Department of Energy National Nuclear Security Administration under Award No. DE-NA0003856, the University of Rochester, and the New York State Energy Research and Development Authority. A portion of this work was performed under the auspices of the U.S. Department of Energy by Lawrence Livermore National Laboratory under Contract No. DE-AC52-07NA27344. The support of DOE does not constitute an endorsement by DOE of the views expressed in this paper. This report was prepared as an account of work sponsored by an agency of the U.S. Government. The views and opinions of authors expressed herein do not necessarily state or reflect those of the U.S. Government or any agency thereof.
[1] S. W. Haan, J. D. Lindl, D. A. Callahan, D. S. Clark, J. D. Salmonson, B. A. Hammel, L. J. Atherton, R. C. Cook, M. J. Edwards, S. Glenzer, A. V. Hamza, S. P. Hatchett, M. C. Herrmann, D. E. Hinkel, D. D. Ho, H. Huang, O. S. Jones, J. Kline, G. Kyrala, O. L. Landen et al., Point design targets, specifications, and requirements for the 2010 ignition campaign on the National Ignition Facility, Phys. Plasmas 18, 051001 (2011).

[2] R. S. Craxton, K. S. Anderson, T. R. Boehly, V. N. Goncharov, D. R. Harding, J. P. Knauer, R. L. McCrory, P. W. McKenty, D. D. Meyerhofer, J. F. Myatt, A. J. Schmitt, J. D. Sethian, R. W. Short, S. Skupsky, W. Theobald, W. L. Kruer, K. Tanaka, R. Betti, T. J. B. Collins, J. A. Delettrez et al., Direct-drive inertial confinement fusion: A review, Phys. Plasmas 22, 110501 (2015).

[3] T. Döppner, D. A. Callahan, O. A. Hurricane, D. E. Hinkel, T. Ma, H.-S. Park, L. F. Berzak Hopkins, D. T. Casey, P. Celliers, E. L. Dewald, T. R. Dittrich, S. W. Haan, A. L. Kritcher, A. MacPhee, S. Le Pape, A. Pak, P. K. Patel, P. T. Springer, J. D. Salmonson, R. Tommasini et al., Demonstration of High Performance in Layered Deuterium-Tritium Capsule Implosions in Uranium Hohlraums at the National Ignition Facility, Phys. Rev. Lett. 115, 055001 (2015).

[4] S. H. Glenzer and R. Redmer, X-ray Thomson scattering in high energy density plasmas, Rev. Mod. Phys. 81, 1625 (2009).

[5] R. G. Kraus, S. T. Stewart, D. C. Swift, C. A. Bolme, R. F. Smith, S. Hamel, B. D. Hammel, D. K. Spaulding, D. G. Hicks, J. H. Eggert, and G. W. Collins, Shock vaporization of silica and the thermodynamics of planetary impact events, J. Geophys. Res.: Planets 117, E09009 (2012).

[6] P. M. Garnavich, B. E. Tucker, A. Rest, E. J. Shaya, R. P. Olling, D Kasen, and A. Villar, Shock breakout and early light curves of type II-P supernovae observed with Kepler, Astrophys. J. 820, 23 (2016).

[7] J. Colvin and J. Larsen, Extreme Physics (Cambridge University Press, Cambridge, 2013).
[8] D. T. Casey, J. A. Frenje, M. Gatu Johnson, M. J.-E. Manuel, H. G. Rinderknecht, N. Sinenian, F. H. Séguin, C. K. Li, R. D. Petrasso, P. B. Radha, J. A. Delettrez, V. Yu Glebov, D. D. Meyerhofer, T. C. Sangster, D. P. McNabb, P. A. Amendt, R. N. Boyd, J. R. Rygg, H. W. Herrmann, Y. H. Kim et al., Evidence for Stratification of Deuterium-Tritium Fuel in Inertial Confinement Fusion Implosions, Phys. Rev. Lett. 108, 075002 (2012).

[9] M. J. Rosenberg, H. G. Rinderknecht, N. M. Hoffman, P. A. Amendt, S. Atzeni, A. B. Zylstra, C. K. Li, F. H. Séguin, H. Sio, M. G. Johnson, J. A. Frenje, R. D. Petrasso, V. Yu. Glebov, C. Stoeckl, W. Seka, F. J. Marshall, J. A. Delettrez, T. C. Sangster, R. Betti, V. N. Goncharov et al., Exploration of the Transition from the Hydrodynamiclike to the Strongly Kinetic Regime in Shock-Driven Implosions, Phys. Rev. Lett. 112, 185001 (2014).

[10] H. G. Rinderknecht, M. J. Rosenberg, C. K. Li, N. M. Hoffman, G. Kagan, A. B. Zylstra, H. Sio, J. A. Frenje, M. Gatu Johnson, F. H. Séguin, R. D. Petrasso, P. Amendt, C. Bellei, S. Wilks, J. Delettrez, V. Yu. Glebov, C. Stoeckl, T. C. Sangster, D. D. Meyerhofer, and A. Nikroo, Ion Thermal Decoupling and Species Separation in Shock-Driven Implosions, Phys. Rev. Lett. 114, 025001 (2015).

[11] H. G. Rinderknecht, P. A. Amendt, M. J. Rosenberg, C. K. Li, J. A. Frenje, M. G. Johnson, H. Sio, F. H. Séguin, R. D. Petrasso, A. B. Zylstra, G. Kagan, N. M. Hoffman, D. Svyatsky, S. C. Wilks, V. Yu. Glebov, C. Stoeckl, and T. C. Sangster, Ion kinetic dynamics in strongly-shocked plasmas relevant to ICF, Nucl. Fusion 57, 066014 (2017).

[12] G. Kagan, D. Svyatskiy, H. G. Rinderknecht, M. J. Rosenberg, A. B. Zylstra, C.-K. Huang, and C. J. McDevitt, Self-Similar Structure and Experimental Signatures of Suprathermal Ion Distribution in Inertial Confinement Fusion Implosions, Phys. Rev. Lett. 115, 105002 (2015).

[13] G. Kagan and X.-Z. Tang, Electro-diffusion in a plasma with two ion species, Phys. Plasmas 19, 082709 (2012).

[14] C. Bellei, H. Rinderknecht, A. Zylstra, M. Rosenberg, H. Sio, C. K. Li, R. Petrasso, S. C. Wilks, and P. A. Amendt, Species 
separation and kinetic effects in collisional plasma shocks, Phys. Plasmas 21, 056310 (2014).

[15] O. Larroche, Ion Fokker-Planck simulation of D- ${ }^{3} \mathrm{He}$ gas target implosions, Phys. Plasmas 19, 122706 (2012).

[16] H. G. Rinderknecht, H.-S. Park, J. S. Ross, P. A. Amendt, S. C. Wilks, J. Katz, N. M. Hoffman, G. Kagan, E. L. Vold, B. D. Keenan, A. N. Simakov, and L. Chacón, Measurements of ion velocity separation and ionization in multi-species plasma shocks, Phys. Plasmas 25, 056312 (2018).

[17] B. D. Keenan, A. N. Simakov, W. T. Taitano, and L. Chacón, Ion species stratification within strong shocks in two-ion plasmas, Phys. Plasmas 25, 032103 (2018).

[18] R. Betti and O. A. Hurricane, Inertial-confinement fusion with lasers, Nat. Phys. 12, 435 (2016).

[19] S. Brygoo, M. Millot, P. Loubeyre, A. E. Lazicki, S. Hamel, T. Qi, P. M. Celliers, F. Coppari, J. H. Eggert, D. E. Fratanduono, D. G. Hicks, J. R. Rygg, R. F. Smith, D. C. Swift, G. W. Collins, and R. Jeanloz, Analysis of laser shock experiments on precompressed samples using a quartz reference and application to warm dense hydrogen and helium, J. Appl. Phys. (Melville, NY) 118, 195901 (2015).

[20] LASL Shock Hugoniot Data, edited by S. P. Marsh (University of California Press, Berkeley, 1980).

[21] R. Cauble, T. S. Perry, D. R. Bach, K. S. Budil, B. A. Hammel, G. W. Collins, D. M. Gold, J. Dunn, P. Celliers, L. B. Da Silva, M. E. Foord, R. J. Wallace, R. E. Stewart, and N. C. Woolsey, Absolute Equation-of-State Data in the 10-40 Mbar (1-4 TPa) Regime, Phys. Rev. Lett. 80, 1248 (1998).

[22] N. Ozaki, T. Sano, M. Ikoma, K. Shigemori, T. Kimura, K. Miyanishi, T. Vinci, F. H. Ree, H. Azechi, T. Endo, Y. Hironaka, Y. Hori, A. Iwamoto, T. Kadono, H. Nagatomo, M. Nakai, T. Norimatsu, T. Okuchi, K. Otani, T. Sakaiya et al., Shock Hugoniot and temperature data for polystyrene obtained with quartz standard, Phys. Plasmas 16, 062702 (2009).

[23] M. A. Barrios, D. G. Hicks, T. R. Boehly, D. E. Fratanduono, J. H. Eggert, P. M. Celliers, G. W. Collins, and D. D. Meyerhofer, High-precision measurements of the equation of state of hydrocarbons at 1-10 Mbar using laser-driven shock waves, Phys. Plasmas 17, 056307 (2010).

[24] S. Hamel, L. X. Benedict, P. M. Celliers, M. A. Barrios, T. R. Boehly, G. W. Collins, T. Döppner, J. H. Eggert, D. R. Farley, D. G. Hicks, J. L. Kline, A. Lazicki, S. LePape, A. J. Mackinnon, J. D. Moody, H. F. Robey, E. Schwegler, and P. A. Sterne, Equation of state of $\mathrm{CH}_{1.36}$ : First-principles molecular dynamics simulations and shock-and-release wave speed measurements, Phys. Rev. B 86, 094113 (2012).

[25] C. Wang, X.-T. He, and P. Zhang, Thermophysical properties for shock compressed polystyrene, Phys. Plasmas 18, 082707 (2011).

[26] S. X. Hu, T. R. Boehly, and L. A. Collins, Properties of warm dense polystyrene plasmas along the principal Hugoniot, Phys. Rev. E 89, 063104 (2014).

[27] S. X. Hu, L. A. Collins, V. N. Goncharov, J. D. Kress, R. L. McCrory, and S Skupsky, First-principles equation of state of polystyrene and its effect on inertial confinement fusion implosions, Phys. Rev. E 92, 043104 (2015).

[28] P. Colin-Lalu, V. Recoules, G. Salin, T. Plisson, E. Brambrink, T. Vinci, R. Bolis, and G. Huser, Dissociation along the principal Hugoniot of the Laser Mégajoule ablator material, Phys. Rev. E 94, 023204 (2016).
[29] T. Döppner, D. C. Swift, A. L. Kritcher, B. Bachmann, G. W. Collins, D. A. Chapman, J. Hawreliak, D. Kraus, J. Nilsen, S. Rothman, L. X. Benedict, E. Dewald, D. E. Fratanduono, J. A. Gaffney, S. H. Glenzer, S. Hamel, O. L. Landen, H. J. Lee, S. LePape, T. Ma et al., Absolute Equation-of-State Measurement for Polystyrene from 25 to 60 Mbar Using a Spherically Converging Shock Wave, Phys. Rev. Lett. 121, 025001 (2018).

[30] S. Zhang, K. P. Driver, F. Soubiran, and B. Militzer, Firstprinciples equation of state and shock compression predictions of warm dense hydrocarbons, Phys. Rev. E 96, 013204 (2017).

[31] S. Zhang, B. Militzer, L. X. Benedict, F. Soubiran, P. A. Sterne, and K. P. Driver, Path integral Monte Carlo simulations of dense carbon-hydrogen plasmas, J. Chem. Phys. 148, 102318 (2018).

[32] C. A. McCoy, S. X. Hu, M. C. Marshall, D. N. Polsin, D. E. Fratanduono, Y. H. Ding, P. M. Celliers, T. R. Boehly, and D. D. Meyerhofer, Measurement of the sound velocity and Grüneisen parameter of polystyrene at inertial confinement fusion conditions, Phys. Rev. B 102, 184102 (2020).

[33] A. L. Kritcher, D. C. Swift, T. Döppner, B. Bachmann, L. X. Benedict, G. W. Collins, J. L. DuBois, F. Elsner, G. Fontaine, J. A. Gaffney, S. Hamel, A. Lazicki, W. R. Johnson, N. Kostinski, D. Kraus, M. J. MacDonald, B. Maddox, M. E. Martin, P. Neumayer, A. Nikroo et al., A measurement of the equation of state of carbon envelopes of white dwarfs, Nature (London) 584, 51 (2020).

[34] S. Zhang, M. C. Marshall, L. H. Yang, P. A. Sterne, B. Militzer, M. Däne, J. A. Gaffney, A. Shamp, T. Ogitsu, K. Caspersen, A. E. Lazicki, D. Erskine, R. A. London, P. M. Celliers, J. Nilsen, and H. D. Whitley, Wide-ranging equations of state for $\mathrm{B}_{4} \mathrm{C}$ constrained by theoretical calculations and shock experiments, in LLE Review Quarterly Report, LLE Review Vol. 164 (Laboratory for Laser Energetics, University of Rochester, Rochester, NY, 2021), pp. 198-200.

[35] J. S. Ross, H.-S. Park, P. Amendt, L. Divol, N. L. Kugland, W. Rozmus, and S. H. Glenzer, Thomson scattering diagnostic for the measurement of ion species fraction, Rev. Sci. Instrum. 83, $10 \mathrm{E} 323$ (2012).

[36] D. Haberberger, A. Shvydky, V. N. Goncharov, D. Cao, J. Carroll-Nellenback, S. X. Hu, S. T. Ivancic, V. V. Karaseiv, J. P. Knauer, A. V. Maximov, and D. H. Froula, Plasma Density Measurements of the Inner Shell Release, Phys. Rev. Lett. 123, 235001 (2019).

[37] A. Shvydky, D. Haberberger, J. P. Knauer, S. T. Ivancic, J. Carroll-Nellenback, D. Cao, I. V. Igumenshchev, V. V. Karasiev, A. V. Maximov, S. P. Regan, P. B. Radha, T. C. Sangster, R. Boni, P. M. Nilson, D. H. Froula, V. N. Goncharov, E. M. Campbell, and V. A. Smalyuk, Shock-release experiments on OMEGA EP (Abstract), in 62nd Annual Meeting of the APS Division of Plasma Physics, Bulletin of the American Physical Society Vol. 65 (American Physical Society, College Park, MD, 2020), Abstract No. NO06.00015.

[38] A. Shvydky, D. Haberberger, A. V. Maximov, R. Boni, D. Cao, J. Carroll-Nellenback, D. H. Froula, V. N. Goncharov, S. X. $\mathrm{Hu}$, I. V. Igumenshchev, S. T. Ivancic, V. V. Karasiev, J. P. Knauer, P. M. Nilson, P. B. Radha, S. P. Regan, J. R. Rygg, T. C. Sangster, M. D. Rosen, and V. A. Smalyuk, Density evolution after shock release from laser-driven polystyrene $(\mathrm{CH})$ targets in inertial confinement fusion, Phys. Plasmas 28, 092703 (2021). 
[39] S. Zhang and S. X. Hu, Species Separation and Hydrogen Streaming upon Shock Release from Polystyrene under Inertial Confinement Fusion Conditions, Phys. Rev. Lett. 125, 105001 (2020).

[40] L. G. Stanton, J. N. Glosli, and M. S. Murillo, Multiscale Molecular Dynamics Model for Heterogeneous Charged Systems, Phys. Rev. X 8, 021044 (2018).

[41] P. A. Amendt, J. L. Milovich, S. C. Wilks, C. K. Li, R. D. Petrasso, and F. H. Séguin, Electric field and ionization-gradient effects on inertial-confinement-fusion implosions, Plasma Phys. Controlled Fusion 51, 124048 (2009).

[42] P. Amendt, S. C. Wilks, C. Bellei, C. K. Li, and R. D. Petrasso, The potential role of electric fields and plasma barodiffusion on the inertial confinement fusion database, Phys. Plasmas 18, 056308 (2011).

[43] P. Amendt, C. Bellei, and S. Wilks, Plasma Adiabatic Lapse Rate, Phys. Rev. Lett. 109, 075002 (2012).

[44] P. Amendt, C. Bellei, J. S. Ross, and J. Salmonson, Ion separation effects in mixed-species ablators for inertial-confinementfusion implosions, Phys. Rev. E 91, 023103 (2015).

[45] D. G. Hicks, T. R. Boehly, J. H. Eggert, J. E. Miller, P. M. Celliers, and G. W. Collins, Dissociation of Liquid Silica at High Pressures and Temperatures, Phys. Rev. Lett. 97, 025502 (2006).

[46] M. J. Rosenberg, F. H. Séguin, P. A. Amendt, S. Atzeni, H. G. Rinderknecht, N. M. Hoffman, A. B. Zylstra, C. K. Li, H. Sio, M. Gatu Johnson, J. A. Frenje, R. D. Petrasso, V. Yu. Glebov, C. Stoeckl, W. Seka, F. J. Marshall, J. A. Delettrez, T. C. Sangster, R. Betti, S. C. Wilks et al., Assessment of ion kinetic effects in shock-driven inertial confinement fusion implosions using fusion burn imaging, Phys. Plasmas 22, 062702 (2015).

[47] H. G. Rinderknecht, P. A. Amendt, S. C. Wilks, and G. Collins, Kinetic physics in ICF: Present understanding and future directions, Plasma Phys. Controlled Fusion 60, 064001 (2018).

[48] H. G. Rinderknecht, D. T. Casey, R. Hatarik, R. M. Bionta, B. J. MacGowan, P. Patel, O. L. Landen, E. P. Hartouni, and O. A. Hurricane, Azimuthal Drive Asymmetry in Inertial Confinement Fusion Implosions on the National Ignition Facility, Phys. Rev. Lett. 124, 145002 (2020).

[49] S. Plimpton, Fast parallel algorithms for short-range molecular dynamics, J. Comput. Phys. 117, 1 (1995).

[50] T. R. Mattsson, J. M. D. Lane, K. R. Cochrane, M. P. Desjarlais, A. P. Thompson, F. Pierce, and G. S. Grest, First-principles and classical molecular dynamics simulation of shocked polymers, Phys. Rev. B 81, 054103 (2010).

[51] T. C. O'Connor, J. Andzelm, and M. O. Robbins, AIREBOM: A reactive model for hydrocarbons at extreme pressures, J. Chem. Phys. 142, 24903 (2015).

[52] S. Root, T. A. Haill, J. M. D. Lane, A. P. Thompson, G. S. Grest, D. G. Schroen, and T. R. Mattsson, Shock compression of hydrocarbon foam to $200 \mathrm{GPa}$ : Experiments, atomistic simulations, and mesoscale hydrodynamic modeling, J. Appl. Phys. (Melville, NY) 114, 103502 (2013).

[53] G. Mogni, A. Higginbotham, K. Gaál-Nagy, N. Park, and J. S. Wark, Molecular dynamics simulations of shock-compressed single-crystal silicon, Phys. Rev. B 89, 064104 (2014).

[54] A. P. Thompson, S. J. Plimpton, and W. Mattson, General formulation of pressure and stress tensor for arbitrary many-body interaction potentials under periodic boundary conditions, J. Chem. Phys. 131, 154107 (2009).

[55] Note that we have removed the regions of $0.5 \mathrm{~nm}$ from the piston and $3 \mathrm{~nm}$ from the location of the shock front when performing the postshock analysis, in order to minimize the errors associated with momentum mirroring by the piston and the viscous shock front.

[56] The larger deviations in $\rho$ than in $u_{\mathrm{s}}$ can be understood by taking the first-order derivative of the jump condition across the shock front [69] $\rho=\rho_{0} /\left(1-u_{\mathrm{p}} / u_{\mathrm{s}}\right)$, which gives $\delta \rho / \rho=$ $1 /\left(1-u_{\mathrm{s}} / u_{\mathrm{p}}\right) \delta u_{\mathrm{s}} / u_{\mathrm{s}}$ For $u_{\mathrm{p}} \leqslant 50 \mathrm{~km} / \mathrm{s}$, we found that the DFTMD-PIMC Hugoniots are well represented by the relation $u_{\mathrm{s}}=$ $1.31 u_{\mathrm{p}}+2.80$. This gives $-3.23<1 /\left(1-u_{\mathrm{s}} / u_{\mathrm{p}}\right)<-1.15$ for $u_{\mathrm{p}} \in[5,50] \mathrm{km} / \mathrm{s}$. Therefore $|\delta \rho / \rho|$ is approximately $(1-3) \times$ $\left|\delta u_{\mathrm{s}} / u_{\mathrm{s}}\right|$, larger for stronger shocks. This derivation also explains why the CMD values of $u_{\mathrm{s}}$ for the same $u_{\mathrm{p}}$ are higher (i.e., $\left.\delta u_{\mathrm{s}} / u_{\mathrm{s}}>0\right)$ if density for the same pressure is lower $(\delta \rho / \rho<$ $0)$ than those given by DFT-MD-PIMC and vice versa.

[57] P. M. Celliers, D. K. Bradley, G. W. Collins, D. G. Hicks, T. R. Boehly, and W. J. Armstrong, Line-imaging velocimeter for shock diagnostics at the OMEGA laser facility, Rev. Sci. Instrum. 75, 4916 (2004).

[58] J. E. Miller, T. R. Boehly, A. Melchior, D. D. Meyerhofer, P. M. Celliers, J. H. Eggert, D. G. Hicks, C. M. Sorce, J. A. Oertel, and P. M. Emmel, Streaked optical pyrometer system for laserdriven shock-wave experiments on OMEGA, Rev. Sci. Instrum. 78, 034903 (2007).

[59] M. C. Gregor, R. Boni, A. Sorce, J. Kendrick, C. A. McCoy, D. N. Polsin, T. R. Boehly, P. M. Celliers, G. W. Collins, D. E. Fratanduono, J. H. Eggert, and M. Millot, Absolute calibration of the OMEGA streaked optical pyrometer for temperature measurements of compressed materials, Rev. Sci. Instrum. 87, 114903 (2016)

[60] A. Stukowski, Visualization and analysis of atomistic simulation data with OVITO-the Open Visualization Tool, Modell. Simul. Mater. Sci. Eng. 18, 015012 (2010).

[61] See Supplemental Material at http://link.aps.org/supplemental/ 10.1103/PhysRevResearch.4.013126 for more postshock results from CMD, discussions about the validity of our scaling approach and effects of rarefaction waves, details of approaches for estimating foil velocities and comparisons with experiments, more comparisons of release profiles with and without thermal expansion, and comparisons between CMD snapshots from different views.

[62] This does not apply to the weaker shock scenario, where chemical bonding is important.

[63] M. Li, H. Zhang, C. Sun, J. Zhao, and H. Yuan, Numerical analysis of laser-driven reservoir dynamics for shockless loading, J. Appl. Phys. (Melville, NY) 109, 093525 (2011).

[64] The thickness of the samples firstly seen through by VISAR can be calculated by integrating the shock velocity over time, which is found to be $56.9 \pm 2.5$ and $44.6 \pm 0.1 \mu \mathrm{m}$ for the weak and the strong shot, respectively. This suggests that the complete $\mathrm{CH}$ sample and only part of the $\mathrm{C}_{50} \mathrm{H}_{48} \mathrm{Br}_{2}$ layer have been seen through when the shock is first observed [labeled as time 2 in Fig. 2(b)], as also evidenced by the wiggles in the left of the shock velocity curves.

[65] Note that in the scaled time, $t=0$ is set to the moment of shock breakout, i.e., $3.1 \mathrm{~ns}$ in the VISAR record shown in Figs. 2(b) and 5 for shot 64755 . 
[66] D. E. Fratanduono et al. (unpublished).

[67] The plasma scale length $s$ is defined by the spatial distribution of plasma density $n_{\mathrm{e}}=n_{\mathrm{e}}^{0} e^{-z / s}$. At $10^{19}$ and $10^{20} \mathrm{~cm}^{-3}$, considering the approximately linear relation between $\log \left(n_{\mathrm{e}}\right)$ and $z$ [Figs. 8(a) and 8(c)], it can be estimated by $s \approx\left[z\left(n_{\mathrm{e}}=10^{19}\right)-z\left(n_{\mathrm{e}}=10^{20}\right)\right] / \ln 10=\left[z\left(n_{\mathrm{e}}=\right.\right.$ $\left.\left.10^{19}\right) / z_{0}-z\left(n_{\mathrm{e}}=10^{20}\right) / z_{0}\right] / 2.3 \times z_{0}$.
[68] We note that the scale length of the hydrogen plasmas is not clearly affected by preheating, as can be seen from the nearly parallel density profiles of hydrogen at every moment shown in Figs. 8(a) and 8(c).

[69] R. P. Drake, High-Energy-Density Physics: Fundamentals, Inertial Fusion, and Experimental Astrophysics (Springer, New York, 2018). 